\title{
Igualdad compensatoria y solidaridad: La propuesta de Pablo Latapí Sarre
}

\author{
Luis Aguilar Sahagún \\ Instituto de Formación Filosófica \\ Intercongregacional, México
}

RESUMEN:

En el presente texto, que forma parte del libro Educación, derechos de la infancia y derecho al desarrollo, se ofrecen elementos de reflexión para proponer una justicia educativa, que supere las insuficiencias del discurso de la "igualdad de oportunidades" y otros similares. Para ello, recurre a las ideas del educador mexicano Pablo Latapi Sarre y en particular, al concepto de "igualdad compensatoria".

\section{ABSTRACT:}

In the following text, which is a chapter of the book Educación, derechos de la infancia $y$ derecho al desarrollo, the author proposes a concept of educative justice, in order to overcome the insufficiencies of the "equal opportunities" discourse and other likeweise. Aguilar Sahagun recovers the ideas of the Mexican educator Pablo Latapi Sarre, and in particular, his concept of "compensatory equity".

\section{Introducción}

La falta de igualdad en educación constituye el aspecto más doloroso del sistema educativo mexicano. Pablo Latapí Sarre ha reconocido este hecho y a lo largo de su larga carrera como investigador de la educación ha propuesto análisis para comprenderlo y darle solución. En su extensa obra ofrece numerosas recomendaciones puntuales. Como él mismo 
reconoce (Latapí: 1993) la problemática que plantea la relación entre justicia y educación tendría que ser abordada en el marco de una teoría de la justicia educativa, terreno poco desarrollado en México. Latapí parte de la constatación de los límites de los enfoques tradicionales sobre el concepto de justicia, incluido, particularmente, el del filósofo John Rawls, quien, con su Teoria de la justicia (1992), ha hecho una de las contribuciones más fecundas de la filosofía política contemporánea. Latapí traza los elementos esenciales de su propuesta a partir de una caracterización de la educación como bien social a distribuir, desde el punto de vista de la igualdad de oportunidades. Este es, sin duda, uno de los puntos más importantes de su reflexión, en el que constata la problemática de este enfoque. Latapí centra su planteamiento bajo el enfoque de la justicia distributiva, es decir, plantea el problema de la política educativa con relación a las decisiones de un poder central o regional que intenta asegurar de forma descendente la distribución equitativa de la educación entre todos los miembros de la sociedad. Si bien este enfoque es importante, es incompleto. Con el fin de superar estas dificultades Latapí busca vincular la idea de igualdad de oportunidades y propone un principio de compensación solidaria y señala modos concretos para hacerlo operativo. En este trabajo nos proponemos mostrar la originalidad de la propuesta de Pablo Latapí, hacer un análisis de sus supuestos, sus alcances, límites y aspectos por desarrollar.

\section{La educación como bien social}

La realización de la justicia educativa supone la distribución equitativa de la educación como bien social que está sujeto a diversas interpretaciones. La primera considera: a) la educación como un sistema independiente y cerrado. Podríamos decir, como una esfera cuyo sentido se agota en sí misma; b) la educación en relación con otros bienes sociales, a los que abre el acceso (mundo del trabajo, cultura, relaciones humanas y sociales más ricas, etc.); c) la educación como una variable más, relacionada con el conjunto de las demás variables sociales y en interacción con ellas. Las implicaciones de la idea de igualdad de oportunidades de educación varían con cada interpretación. "La complejidad sociológica que brota de estas alternativas — señala Latapí- es también una complejidad filosófica, pues en cada escenario varía la definición del bien cuya distribución se plantea” (Latapí: 1993, p. 23). 
Con el fin de lograr una caracterización más diferenciada de la educación como bien social, Latapí destaca los siguientes rasgos:

a) A diferencia de los demás bienes sociales, la educación se asimila al sujeto, lo transforma, se vuelve parte constitutiva de su ser. De ahí que sólo pueda hablarse de distribuir la educación en un sentido análogo y, en realidad, muy problemático. Latapí señala una característica de particular relevancia respecto de la educación: su efecto en las personas produce transformaciones desiguales. Es decir, la educación es generadora de desigualdades. Esta es otra de las paradojas de la justicia educativa, cuya trascendencia no suele ser suficientemente percibida. Si bien es imposible renunciar al ideal de un sistema educativo que ofrezca la misma calidad, tanto las condiciones de la realidad social de nuestro país como las enormes diferencias en la oferta educativa de instituciones públicas y privadas, no se puede perder de vista que la oferta educativa es, de hecho, generadora de desigualdades sociales que puede llegar a polarizar aún más sus extremos. Recíprocamente, la calidad de la oferta educativa tiene una de sus raíces en las enormes deficiencias sociales existentes. La doble relación entre causa y efecto no es biunívoca.

b) Hay personas más o mejor educadas que otras: el acento de lo que constituye la calidad de la educación puede ponerse en determinados valores humanos, en destrezas, habilidades y competencias. En general, suele entenderse que una persona mejor educada es más capaz de abrirse camino en la vida y de vivir bien, de acuerdo con una concepción de la buena vida que puede diferir dentro de un amplio espectro de posibilidades, pero que tiene como base común lo que solemos entender por condiciones indispensables de una vida digna.

c) La calidad de la educación tendría que incluir como uno de sus componentes esenciales el desarrollo de la conciencia ciudadana y del sentido de responsabilidad social. De faltar este factor sería más preciso hablar de mujeres y hombres habilitados o altamente capacitados para ejercer un oficio o una profesión, pero no de personas bien educadas en sentido estricto.

d) La educación tiene un carácter difusivo, se extiende a otras personas, grupos e instituciones a través de los sujetos que la han asimilado. La persona educada enriquece la convivencia. Podemos decir que este enriquecimiento se puede dar a dos niveles. El primero es el 
efecto expansivo que tiene de suyo la educación, de modo intencionado o no. La sociedad en su conjunto sale ganando por contar con personas más o mejor educadas que otras. El segundo nivel implica la determinación consciente, intencional por parte del sujeto, de darle a su actividad un sentido por el que busque de modo intencional contribuir a una mayor humanización de la sociedad y a un auténtico progreso.

e) Un nivel de educación elevado y extendido de modo significativo dentro de una sociedad enriquece la cultura, plantea nuevas exigencias y modifica las costumbres en sentido positivo. Si se ignoran estos efectos de la educación, la justicia educativa se reduce al problema de la distribución equitativa de oportunidades educativas, planteamiento que resulta insuficiente. Esto permite ver que la educación no puede reducirse a ser un mero medio de movilidad social. Tampoco se puede perder de vista que, dada a partir de la naturaleza misma del bien asimilado, la equidad educativa está asociada a un conjunto de obligaciones que se traducen en distintos modos de aportar al todo social: por lo que llega a ser, lo que sabe hacer, los bienes que puede compartir, etc.

f) El punto de partida de la educación, las preferencias individuales, el entorno social y educativo y, particularmente, el esfuerzo personal de cada educando, son factores muy variables que plantean una dificultad enorme para dar sentido a la exigencia de una igualdad de oportunidades aplicable a cada caso particular.

La comprensión de la educación como un bien intermedio, por encima del mínimo de los bienes fundamentales no está exenta de dificultades teóricas y prácticas. La educación está en estrecha correlación con el acceso a los bienes fundamentales, tales como un mínimo de alimentación o de recursos económicos.

En el lenguaje de la tradición del pensamiento social cristiano se habla del destino universal de los bienes. Al añadir que se trata de los bienes fundamentales — que es necesario especificar- Latapí añade un matiz de importancia. No todos los bienes tendrían un destino universal. A pesar de su aparente evidencia, este principio plantea problemas de interpretación que será necesario clarificar. 


\section{Los principios de igualdad compensatoria y de proporcionalidad solidaria}

Si bien Latapí constata que aún está por construirse una teoría de la justicia educativa aplicable a México (Ídem, p. 32), propone algunos elementos teóricos y normativos a partir de los cuales se podría elaborar una teoría más completa que pueda ser sometida a una discusión colectiva. La igualdad, la libertad, la justicia y la democracia son valores que se derivan de la dignidad de la persona. La libertad es entendida aquí como la capacidad de hacer opciones responsables; la igualdad supone el acceso a los puestos y cargos públicos abierto a todos los ciudadanos; la justicia supone la existencia de normas que regulen la distribución de los beneficios y las oportunidades sociales; la democracia exige la constitución de un gobierno representativo.

Latapí explora las posibilidades del sentido de hablar de justicia educativa en términos de un bien a distribuir. Al describir los rasgos fundamentales del amplio espectro de dimensiones abarcado por el concepto de educación, constata lo problemático de este enfoque. No obstante, propone criterios de carácter compensatorio que vienen acentuadas bajo el ángulo de la justicia social. Atento a la complejidad de la educación como bien para la persona y para la sociedad, su propuesta abarca tanto criterios que atienden al rendimiento como a la necesidad, todo bajo el supuesto del principio de igualdad compensatoria (PIC). Dada la imposibilidad de hacer justicia atendiendo a un solo criterio, el PIC combina el rendimiento y la necesidad. Para ello Latapí se basa en presupuestos que superan la concepción de la justicia de rendimiento. La justicia distributiva supone un criterio de asignación en correspondencia con la naturaleza de los bienes a distribuir, como con la organización social necesaria para llevarla a cabo. En este contexto, cobra sentido el principio de proporcionalidad solidaria (PPS), que regula la distribución de los bienes que desbordan el ámbito de los bienes que pueden ser considerados como fundamentales, por constituir el mínimo indispensables para la realización de la persona (como la alimentación, un mínimo de recursos económicos). Este principio establece que la magnitud de la apropiación particular de un bien fundamental debe guardar proporción con la distribución existente de ese bien en esa sociedad determinada (Latapí, ídem, p.34). El bien social de la educación se ubica entre los bienes que están por encima del mínimo señalado. Este principio regularía los

\section{3}


límites de apropiación o acceso a un bien cuya posesión se justifica por el talento, el esfuerzo y, tomando en cuenta que todos los miembros de la colectividad tendrían que poder disfrutar de él, en la herencia. El principio de proporcionalidad solidaria tiene un triple fundamento: a) el llamado destino universal de los bienes fundamentales; b) la igualdad básica de todos las personas y c) la solidaridad con quienes se comparte un destino común, que remite al principio de solidaridad, uno de los pilares de la Enseñanza social de la Iglesia católica (Cf. Nell-Breuning: 1985; 1994; Kerber: 1996; Calvez: 1991).

La ubicación de la educación como un bien intermedio, entre los bienes fundamentales y los bienes de carácter suntuario, no está exenta de dificultades teóricas y prácticas. La educación está en estrecha correlación con el acceso a los bienes fundamentales, tales como un mínimo de alimentación o de recursos económicos. En el lenguaje de la gran tradición del pensamiento social cristiano se habla del destino universal de los bienes. Al añadir que se trata de los bienes fundamentales — que sería necesario especificar - Latapí añade un matiz de importancia. No todos los bienes tienen un destino universal. A pesar de su aparente evidencia, este principio plantea problemas de interpretación que será necesario clarificar.

La validez del alcance práctico del PIC descansa en tres pilares básicos que conviene analizar por separado: A) La dignidad de la persona; B) El destino universal de los bienes y C) la solidaridad en un destino común.

A) La dignidad de la persona. Dado que la justicia es ante todo un imperativo de conciencia y un anhelo de toda persona, este principio establece la primacía del orden moral sobre el orden jurídico. Sobre este criterio descansa una de las tradiciones más antiguas que dan sustento a los derechos humanos. Se trata de una idea de múltiples raíces históricas que dentro de la tradición occidental cobró particular fuerza a partir de la filosofía griega, la teología cristiana y el derecho romano. A partir de la revolución francesa la idea de dignidad humana quedó consagrada como pilar de un humanismo secular dentro del cual los derechos humanos encontraron su sustento común. La raíz de la dignidad humana como un valor preeminente sobre todas las cosas y como un fin en sí mismo ha encontrado diversas formulaciones ético filosóficas en las que se ha intentado destacar la igualdad de todos los seres humanos. La igualdad se refiere ante todo al valor de la persona y de su libertad, 
precisamente por tratarse de un ser consciente con capacidad de desarrollarse en libertad (Cf. Álvarez González: 1999).

- La idea de dignidad encierra algunas dificultades. Por una parte, el reconocimiento del valor de personas libres e iguales sólo se puede sustentar en la autonomía moral del sujeto (Kant). Pero últimamente, ésta postula un fundamento trascendente, cuya existencia se ha sometido a discusión dentro de una sociedad pluralista y secular.

- Por otra parte, el concepto de dignidad de la persona puede quedar vaciado de todo contenido vinculante fuera de un sistema de derecho en el que las leyes tienen un fundamento en la justicia objetiva. Resulta discutible vincular todos los derechos humanos y todas las exigencias que se reivindican en su nombre sobre la base de un fundamento de alcance prácticamente ilimitado. Su sola postulación o invocación no son suficientes. Sólo es posible determinar su contenido históricamente, en relación con las experiencias históricas de las diversas sociedades políticas.

B) La idea de un destino universal de todos los bienes aparece en muy distintos autores y fundamentada de manera diferente por cada uno de ellos. La concepción original de esta idea es de orden teológico y parte de la comprensión del mundo como creación divina, en la que los bienes de la tierra han de servir a todos los seres humanos para que alcancen su desarrollo. En los autores clásicos como Locke, Hobbes, Rousseau, e incluso Marx, volvemos a encontrar esta idea bajo una formulación distinta, en un contexto secular.

- La adopción que hace Latapí de este principio descansa en la tradición cristiana, concretamente, en la formulación elaborada por Tomás de Aquino y adoptada y desarrollada en el corpus del magisterio eclesial conocido como Enseñanza social de la Iglesia católica (Cf. Calvez: 1991). En esta doctrina, el presupuesto básico es la afirmación del mundo como creación de un Dios único, tal como se describe en el pensamiento bíblico. Hablar de destino universal es señalar una finalidad asignada al conjunto de todos los bienes creados. Afirmar que Dios creó todo para todos puede resultar plausible desde el punto de vista de una Teología que corre el riesgo de no tener suficientemente presente la dimensión histórica de la acumulación y distribución de los bienes. En su versión secular este principio ha sido reconstruido por autores clásicos como Locke, Rousseau o Marx, que han recurrido a reconstrucciones imaginarias de este

\section{5}


proceso en una perspectiva evolutiva (desposesión original, acumulación original, etc.) que han conducido a la ficción de un contrato social que funda la comunidad política. Como señala Guitton (1956) la idea del contrato social es producto de un pensamiento que tiende a disociar el todo estructural de la realidad social en entes ideales atomizados, ciudadanos y ciudadanas plenamente conscientes de su valor inalienable como personas, que proceden de acuerdo con procedimientos e instituciones diseñadas por una supuesta razón pura. Este tipo de pensamiento prepara un régimen político en el que sería posible una estructura política que fuera a la vez racional y natural, resultado de un consenso colectivo logrado espontáneamente.

- La exigencia de que la apropiación privada de los bienes económicos y de los que se relacionan íntimamente con ellos tenga una función social, encuentra su única justificación en relación con el PPS. Este principio supone la unidad indisoluble de los valores de la justicia y de la caridad, tal como la ha mantenido la teología moral cristiana.

- Cabe notar que el principio mencionado cobra eficacia y plausibilidad sólo dentro de un clima social en el que la tradición cristiana siga permeando de forma significativa el horizonte de autocomprensión de la mayoría de sus miembros. Cuanto más liberal se vuelve una sociedad, tanto mayor es el riesgo de que el principio pierda la base que le dé sustento real o quede como consigna de carácter puramente declaratorio.

- La justificación de las diferencias en la distribución y apropiación de ciertos bienes sociales, así como la regulación de las compensaciones orientadas a equilibrarlas, tienen su fundamento en el PPS. Latapí advierte que, por su carácter general, este principio no ofrece reglas precisas de distribución. Ésta sería una tarea propia del derecho positivo. Como hemos señalado más arriba, en este campo existen avances significativos.

- Aun cuando el sentido de la justicia puede captar el significado de expresiones que intentan traducir la idea del destino universal de los bienes en términos de sentido social de la propiedad privada o de bipoteca social de los bienes poseídos, resulta problemático concretizar sus implicaciones concretas. En buena medida, el alcance de su sentido es tan profundo como la comprensión que cada persona logra de su propia relación con el mundo y de su vínculo con los demás. 
- El principio del destino universal de los bienes funciona en todo caso como una idea regulativa de carácter formal, pero de gran alcance en sus consecuencias. Su aceptación supone la buena fe, y conduce a relativizar el sentido de la posesión de lo tuyo y de lo mío. El principio abre un horizonte de distribución, redistribución, don y comunicación de bienes, cuyo límite sólo pueden establecerlo quienes lo adopten como principio ético general, pero adolece de fuerza vinculante como elemento clarificador de los criterios normativos concretos de distribución y redistribución de los bienes.

C) Solidaridad en un destino común. Este enunciado es tanto un enunciado descriptivo como normativo y constituye el fundamento del principio de solidaridad (Cf. Nell-Breuning 1990; Höffe 1999, pp. 189-194). De acuerdo con este principio, cada persona, grupo o comunidad ha de contribuir al bien común de la comunidad política según sus posibilidades, y de acuerdo con los diferentes estratos del bien común; y tiene derecho a recibir de los demás todo lo que necesita para vivir y desarrollarse, si por justas causas él o ella no puedan por sí mismos tener acceso a lo que necesitan. A este derecho corresponde una obligación que, según sea el caso, toca a los padres, a la familia, a las asociaciones intermedias y finalmente al Estado.

- El principio de solidaridad tiene por objeto, en primer lugar, el bien común, y la obligación de contribuir a él; es un deber de justicia que cada persona debe cumplir según su propia capacidad y en atención a la necesidad ajena, promoviendo y ayudando a las instituciones tanto públicas como privadas. La solidaridad está vinculada a los valores de justicia, libertad, igualdad, participación, etc.; expresa la condición ética de la vida humana común, y tiene como acción suprema el compartir y la participación. Todos los hombres y mujeres tienen derecho a los valores humanos, como la verdad, la libertad, el desarrollo, la justicia, la paz, etc. Sería falso interpretar el principio de solidaridad como un pretexto para no asumir las propias responsabilidades personales, o para abusar de los bienes y servicios comunes (García de Alba: 1999, pp. 191-192).

- El interés común de una colectividad se plantea como el fin que ha de orientar la actividad individual y las decisiones en materia de política social. Su formulación como bien común, entendido como principio normativo de las políticas de distribución y redistribución supone un análisis crítico de su contenido y de su significado en relación con otros elementos constitutivos de este concepto. ${ }^{1}$ 
- La expresión política del principio de solidaridad es la democracia radical como modelo de asociación para la libertad y la igualdad (Cf. Villoro, 1998, cap. 16). Su fundamento es la idea de fraternidad y supone la existencia de una auténtica comunidad. En ella, cada individuo asume la prestación de un servicio a la colectividad. La comunidad "es el conjunto de relaciones serviciales, en que cada quien da algo de sí, y no la sola sumisión a la ley común" (Villoro, op. cit., p. 361). La base de la comunidad es la reciprocidad de dones.

\section{Concretizaciones}

De la aplicación de los principios de igualdad compensatoria y de proporcionalidad solidaria se seguirían las siguientes consecuencias prácticas:

1. Igualdad de acceso y permanencia de una educación básica que corresponde al Estado garantizar y ofrecer de forma gratuita. La determinación del grado de educación básica que ha de garantizar el Estado, es una cuestión que queda abierta al debate público y que ha de tomar en cuenta una gran cantidad de factores. Como medio concreto para garantizar la realización de este principio entre los más necesitados, el Estado tiene la obligación de otorgarles subsidios y becas para los gastos indirectos. Resulta claro que el Estado no puede garantizar la igualdad de permanencia, sino a lo sumo un mínimo de condiciones estructurales que al menos no la impidan.

2. La política educativa debe tener como criterio la igualdad de insumos, en cantidad y en calidad, en todos los establecimientos de enseñanza básica. Como medio para garantizar el funcionamiento de este principio, el Estado puede exigir que todos las escuelas funcionen de acuerdo con estándares mínimos que involucra al profesorado, al alumnado y a las madres y padres de familia. Este ideal resulta particularmente difícil de alcanzar, sobre todo respecto de la calidad de los insumos. Por eso se hace imprescindible un tercer elemento.

3. La compensación de insumos. Esto supone la superación del criterio de igualdad por el de equidad. Si se toma como base la racionalidad de la proporcionalidad solidaria, los mejores insumos han de ser destinados a las escuelas de las zonas más atrasadas y pobres del país, con el fin de fortalecer a los más débiles. Este modo de proceder tiene el propósito de que la asignación de los recursos educativos no sea una causa del incremento de las desigualdades sociales, sino que por el contrario, 
contribuya a que disminuyan. Es importante que las políticas compensatorias jamás pierdan de vista la naturaleza de la educación en toda su amplitud.

\section{Balance y conclusiones}

Podemos concretizar lo que nos parecen ser los límites y los alcances de la propuesta de Pablo Latapí en los siguientes puntos:

1. La peculiaridad de la naturaleza del bien social de la educación hace muy compleja la determinación del significado de la justicia en esta materia y por lo tanto de la valoración de las políticas orientadas a promoverla. Por eso, la obligación del Estado respecto a la justicia educativa ha de ser probablemente más modesta de lo que podemos suponer o querríamos exigir. Concretamente, el principio de igualdad de oportunidades da lugar a tal cantidad de equívocos que resulta de poca utilidad, si en la adopción de políticas y en la reivindicación de derechos no se hacen las aclaraciones pertinentes en cada caso. La igualdad buscada no resulta automáticamente de tratar igual a los desiguales, de identificar a los grupos más desfavorecidos y de aplicar medidas compensatorias. Latapí señala un aspecto indispensable, que complemente e incluso puede tener mayor eficacia en el diseño y la adopción de políticas educativas que generen mayor equidad: claridad en la definición de los conceptos, de los objetivos, los diagnósticos, las estrategias; rigor en la fundamentación de los programas; la finura del análisis y el profesionalismo en la experimentación y en la investigación (Latapí: 1995, p. 63).

2. Latapí propone los elementos de una teoría de la justicia educativa adecuada al bien social a "distribuir", tomando en cuenta que la concepción de la justicia entendida como igualdad de oportunidades se topa con graves dificultades, tanto de clarificación conceptual como de aplicación práctica. Esto pone en evidencia que la idea de igualdad de oportunidades es legítima sólo bajo la estricta delimitación de los ámbitos en los que se exige y del conjunto de bienes educativos implicados en cada caso. Además, hablar de distribución de un bien alude de forma directa a criterios de justicia distributiva asociada al rendimiento como criterio de asignación, mientras que la exigencia de igualdad de oportunidades es una de las formas de entender la idea de justicia social. Si no se establecen los límites formales entre una y otra concepción se puede incurrir en confusiones y, por lo mismo, en grandes dificultades para su aplicación. 
3. La sobreposición de ideas tiene su origen, muy probablemente, en el hecho de que la educación se ha consignado ya como objeto de derecho positivo. Esto es, sin duda, un logro de reivindicaciones sociales orientadas por una determinada idea de la igualdad social o, lo que es lo mismo, de una concepción de lo que debe de ser una sociedad igualitaria. Una vez consignado como derecho positivo, se espera que la distribución de los bienes que garantizan su vigencia sea justa, igualitaria o equitativa. Si bien el uso que se hace de estos términos deriva su significado parcial de una determinada ideología (por ejemplo, la que se gestó durante la revolución mexicana), su significado real se desprende de la situación concreta en que se encuentran millones de niños, niñas y personas adultas en materia educativa: analfabetismo real o funcional, bajos grados de escolaridad, bajos rendimientos, baja calidad en el desempeño profesional, disparidades extremas en el acceso a los bienes educativos, etc.

4. La justicia educativa tiene su fundamento en el derecho a la educación. Sin embargo, como ya hemos visto, la naturaleza de este bien social — como un bien necesario para la realización de la persona; como un medio de acceso a otros bienes y oportunidades y como un instrumento de cohesión social y un bien para el Estado mismo- hace necesario que en su distribución se busque conciliar los intereses de quienes se benefician de él: la persona, la sociedad y el Estado.

5. Como bien fundamental, la distribución de los bienes asociados a la educación debería estar sujeta al principio de proporcionalidad solidaria, cuya aplicación debería estar garantizada por el Estado. No se puede exigir que los sistemas educativos contrarresten completamente las desigualdades que se generan dentro de una sociedad a causa de los factores ajenos a la educación. El límite de la responsabilidad por parte del Estado se puede precisar a partir de un conjunto orgánico de normas articuladas de acuerdo con la racionalidad que supone la proporcionalidad solidaria. La similitud formal que presentan con algunas de las interpretaciones del principio de igualdad de oportunidades no es relevante. Con lo que se confirma que, dada la cantidad de interrogantes que deja sin resolver, esta exigencia constituye más bien un ideal programático o idea regulativa que un principio normativo en sentido estricto. Podemos decir que si bien la idea de igualdad de oportunidades deja sin resolver las paradojas y aporías que plantea la aplicación de la justicia en el 
ámbito educativo, cumple al mismo tiempo un gran servicio tanto en el orden práctico como teórico. Al ponerlas de relieve, manifiesta los límites de una exigencia, la complejidad de sus alcances y la dificultad de su cumplimiento.

6. Una teoría de la equidad educativa ha de poder establecer con claridad cuáles son los bienes que tendrían que estar al alcance de toda persona, independientemente del contexto socioeconómico en el que se ubique. Entendidos de esta manera, los bienes que, en la representación social de cada cultura, al menos en cuanto a la oportunidad de acceder a ellos, son considerados como indiscutiblemente necesarios para el desarrollo de toda persona, constituyen el bien común de esa sociedad. El camino para lograrlo no consiste en la deducción de principios abstractos, sino en el discernimiento que se realiza a partir de un análisis cultural y político que da sustento y vida a una sociedad.

7. Entre esos bienes irreductibles se encuentra la educación, particularmente la de la infancia. Garantizar el derecho al desarrollo educativo de la infancia implica garantizar las condiciones materiales del aprendizaje.

8. En sociedades como la nuestra, tan polarizada en recursos y oportunidades reales de acceso a una mejor educación, es necesario que el Estado establezca algún tipo de sanción social a quienes gozan del tipo de educación que Latapí caracteriza como opulencia educativa, que puede ser entendida como el acceso irrestricto a todo tipo de bienes educativos que se sitúan muy por encima de los bienes indispensables asociados a la educación básica. Con este término Latapí destaca un aspecto importante de la educación como bien social: los aspectos de carácter suntuario que la pueden acompañar deben compensarse políticamente para garantizar un mínimo de homogeneidad social. Aquí podría incluirse una larga lista: desde la orientación curricular de algunas carreras, textos de poca relevancia educativa, posgrados o especialidades con poca o nula trascendencia social, etc.

9. Como objeto de distribución, la educación está asociada a un mínimo de financiamiento y de múltiples bienes educativos: libros, materiales de trabajo, espacios adecuados, medios informáticos, magisterio cualificado, etc. Muchos de estos bienes son imponderables. Sin un sustento económico básico y sin alternativas de educación es imposible el ejercicio de la libertad.

\section{1}


10. La definición de políticas orientadas a la equidad educativa se ha de diferenciar según se trate de educación básica (preescolar, primaria, secundaria, media-superior o superior. La atención a esta diversidad de aspectos bajo la perspectiva de los derechos de la infancia nos conducirá a conclusiones aún más matizadas. Nuestro objetivo es complementar el concepto de equidad educativa bajo el punto de vista de la justicia social.

11. Entre las medidas que menciona Latapí para que una política social orientada a la equidad sea algo más que el mero combate contra la pobreza en todas sus manifestaciones (que es, en el fondo, un modo de asistencialismo), interesa destacar la destinación del gasto social intencionalmente compensatorio en el ámbito educativo, sin desligarla de todas las demás medidas políticas, culturales y económicas que han de integrar un buen plan de desarrollo: medidas fiscales efectivamente redistributivas, mejoramiento de salarios mínimos, reestructuración del sector salud, promoción del arte y la cultura; protección y promoción de los derechos de los más débiles y marginados en todos los campos, etc. El efecto compensatorio de estas medidas podría impedir que sigan creciendo las excesivas desigualdades existentes e incluso que comiencen a disminuir progresivamente. Esta expectativa no es concebible sin una teoría de la justicia en la que los efectos favorables de una economía de mercado contribuyan a ampliar las expectativas de los que se encuentran en mayor desventaja. "Una auténtica preocupación por la justicia se revela en medidas que aumenten las expectativas de equidad de las mayorías". ${ }^{2}$ El problema de fondo sólo se resuelve sobre la base de una filosofía política adecuada, no de una razón estratégica, tecnológica o instrumental. Aún carecemos de una filosofía a la altura de esta problemática.

\section{BiBLIOGRAFÍA}

AA.VV., La democracia y los derechos sociales y económicos (1999), México, Asociación Mexicana de las Naciones Unidas/Universidad Autónoma de Aguascalientes

Álvarez González, A.: 1999, Hacia una teoría crítica de la dignidad de la persona, Universidad de Alcalá, Alcalá 
Calvez, Jean-Yves (1991). Enseñanza social de la Iglesia, Barcelona.

Delors, Jacques (1996). La educación encierra un tesoro, Santillana/UNESCO, Madrid.

Flores Rentería, Joel "Derechos diferenciados: ¿una vieja versión de la justicia?”, en Política y cultura, Cerano 1999, No 12, Universidad Autónoma de Xochimilco, México.

Galbraith, John K. (1997), Una sociedad mejor, Madrid.

Latapí, Pablo, "Reflexiones sobre la justicia en la educación" en Revista Latinoamericana de Estudios Educativos, Vol. XXIII, No. 2, 1993.

, "El derecho a la educación y a la educación superior" en Gutiérrez Gallo, Amador y María Matilde Martínez Benítez (compiladores) 1999.

de Aguascalientes, Aguascalientes.

Heller, Agnes (1994). Más allá de la justicia, Crítica, Madrid.

Höffe, Otfried (Coord.) (1992). Wörterbuch der Ethik (1992), Beck, Münich.

Frankfort.

(1999) Demokratie im Zeitalter der Globalisierung, Beck,

Rawls, John, $\left(199212^{\mathrm{a}}\right)$. A Theory of Justice, Universidad de Cambridge, Massachusetts.

Frankfort.

(1994) Die Idee des politischen Liberalismus, Suhrkamp,

bridge, Mass.

(1999) Collected Papers, Harvard University Press, Cam-

Ricoeur, Paul (1999). Lo justo, Caparrós, Madrid. 


\section{Notas}

1 En la tradición liberal se emplea el concepto de "interés colectivo" como correlato del concepto de bien común, de cuño iusnaturalista. Sin embargo, dentro del liberalismo actual han intentos de recuperar el sentido sustancial de bien común. Cf. Nell-Breuning, Oswald von (1957) Lexikon für Politik, Friburgo, pp. 42 y ss.; Calvez, Jean-Yves (1991) Enseñanza social de la Iglesia, Barcelona; Kauffmann, Arthur (1994), Negativer Utilitarismus, Ein Versuch über das Bonum comune, Munich, pp. 8-29; Valadier, Paul (1980), Agir en politique, Décision morale et pluralisme politique, París, pp. 121-135; Schumacher E. F., Es gebt auch anders, Jenseits des Wachstums (1974), Munich, pp. 12 y ss; Rawls, John (1994), Die Idee des politischen Liberalismus. Aufsätze 1978-1989, Francfort. Jordan, Bill (1989), The common good, Citizenship, morality and self interest, Nueva York, pp. 108-126). El problema central del concepto de bien común puede plantearse bajo la cuestión de cómo comulgar en una idea de la Justicia (cfr. supra). En otro trabajo hemos analizado la problemática que plantea este concepto Cf. Aguilar, Luis Armando: "Pluralismo y bien común: posibilidades en tensión en el México actual", en Logos. Revista de Filosofía, No 86, Mayo-Agosto de 2001, Guadalajara, pp. 45-59.

$2 \quad$ Ídem., p. 132 